\title{
Computer-Assisted Analysis of Transrectal Ultrasound Images
}

\author{
Brian L. Craine, Gregory Oldani, John R. Engel, Ronald F. Whitney, David Wright, and Eric R. Craine
}

\begin{abstract}
We have developed a microcomputer based system with an application specific software package which permits the direct digitization and analysis of transrectal ultrasound (TRUS) images. The system is highly flexible and enables access to a wide range of image analysis tools through relatively simple software modifications, which cannot be implemented using a standard ultrasound instrument. We have demonstrated the capability of the system by an analysis of a number of morphometric parameters and by a correlation of these measurements with the presence of prostatic cancer. We found that the measurement of the ratio of the anterior-posterior axis to transverse axis and the presumed circular area ratio (PCAR) were significent predictors of prostatic cancer. The sensitivity of the PCAR measurement was $93 \%$, the specificity was $50 \%$, the positive predictive value was $57 \%$, and the negative predictive value was $91 \%$. The high negative predictive values of these parameters may provide objective criteria to allow for selective biopsy of patients. This system provides researchers with an efficient, economical, and flexible method to aid in the analysis of TRUS images in a quantitative manner.

(C) 1990 by W.B. Saunders Company.
\end{abstract}

KEY WORDS: image analysis, transrectal ultrasound, prostatic cancer.

$\mathbf{T}$ HE EVALUATION of the prostate gland is a major medical problem. Prostate carcinoma is the second leading cause of cancer deaths in males. Most recent estimates are that 28,000 men would die from prostate cancer in the United States in 1988 and that there would be approximately 99,000 new cases diagnosed. ${ }^{l}$ Autopsy results indicate the prevalence of carcinoma may be as high as $15 \%$ to $40 \%$ of males older than 50 years of age. ${ }^{2,3}$ In addition, it is estimated that as many as $80 \%$ of older males have benign prostatic hypertrophy. ${ }^{4}$ The diseases of the prostate are age related, and the shifting demographics of this country to much older population insures that the numbers of patients with prostatic disease will be increasing in the future. Indeed, the trend of the incidence of prostate cancer as reported by the US National Center for Health Statistics has been increasing since $1960{ }^{1}$

Success in the treatment of prostate carcinoma will no doubt increase because of early diagnosis. It is particularly important to detect the disease while it is operable and before it metastasizes to other parts of the body. Occult prostatic cancer has no specific clinical indications. The use of biochemical markers to indicate the presence of prostate carcinoma has been evaluated ${ }^{5}$; in particular, blood serum levels of prostatic specific antigen (PSA), acid phosphatase, and alkaline phosphatase have been measured and found to be elevated in patients with carcinoma of the prostate. Unfortunately, patients with benign prostatic hypertrophy also have elevated levels of these markers, which markedly decreases the specificity of the tests. This is especially true since the population needing screening the most has an extremely high incidence of benign prostatic hypertrophy (approaching $80 \%$ ).

A malignancy may be suspected by the physician when the digital rectal exam reveals a palpable hard area. While it was once thought that the majority of prostatic cancers were located posteriorly in the region most accessible in a clinical examination, it has now been shown that this is not true. This was a false clinical impression since these were the cancers most commonly detected. $\mathrm{McNeal}^{6}$ has shown that the cancer develops in all regions of the peripheral tissue with equal likelihood. The development of cancers in the lateral peripheral zone and transition zone are anteriorly located and are, therefore, more difficult to detect clinically. Niternative modes of diagnosis are clearly needed.

Evaluation of the prostate by ultrasound has been found to be very useful. While $\mathrm{x}$-ray studies reveal little of the structures of the prostate, transrectal ultrasonagraphy produces excellent images of the prostate. The use of the transrectal ultrasound allows for the evaluation of all regions of the prostate. Studies indicate that use of the transrectal ultrasound allows for the detection of clinically significant cancers at a frequency about double that obtained by a physical exam alone.?

From Western Research Company, Inc, Highland General Hospital, Oakland, CA, and Prescott Urology, Ltd.

Address reprint requests to Dr Brian Craine, Western Research Company, Inc, 85 Bolinas Rd, Suite 18, Fairfax. CA 94930 .

- I990 by Society of Photo-optical Instrumentation Engineers.

$0897-1889 / 90 / 0304-0005 \$ 03.00 / 0$ 
Thus, the use of transrectal ultrasound in the evaluation of the prostate serves several roles including identifying nonpalpable tumors; staging known disease; directed biopsy of suspected tumors; directed placement of radioactive seeds for treatment; and determining glandular volume.

In order to improve the diagnostic accuracy and ease of interpreting transrectal ultrasound (TRUS) images, Kimura et $a^{8}$ defined some easily recognized features that could be quantitatively and objectively measured. These measurements were then used to predict the disease status of the patient. These geometrical parameters were chosen because of the long recognized characteristic changes that occur in prostatic cancer described by Watanabe et $a^{9}$ as early as 1976. These include changes in the anteriorposterior diameters, changes in the superiorinferior diameter, irregularity of transverse section shape, changes in section shape from level to level, and loss of symmetry. However, the measurement of these parameters is not conveniently accomplished with the current ultrasound units. Kimura et $\mathrm{al}^{8}$ photographed TRUS images and used projected images on a digitizing board to obtain the measurements. This method is not feasible in usual practice.

We have developed an inexpensive hardware/ software system to enhance the utility and flexibility of the TRUS instrument. Our system consists of a dual buffered frame grab board which is connected directly to the ultrasound instrument, providing computer control over the images produced. Because software is more easily modified than hardware, our system allows for a more flexible analysis of the TRUS images than could be accomplished with most existing ultrasound instruments. These analyses could, for example, include image processing, morphometric measurements, or feature recognition.

To demonstrate the added flexibility our system affords, we report here the development and testing of a software package to conveniently collect and organize a series of geometric measurements from prostatic ultrasound images. We then correlate these measurements with the diagnosis of prostatic cancer. The software package, PROST (Tucson, AZ), is designed to simplify the acquisition of geometric parameters characterizing the prostate gland, and to analyze that data in a fashion introduced by Kimura et al. ${ }^{8}$

\section{MATERIALS AND METHODS}

\section{Description of Hardware/Software System}

We have developed an inexpensive computer operated system which allows for the direct acquisition and analysis of the images obtained with a TRUS. The system is based on an 80286 host microcomputer equipped with a Western Research Company, Inc, (Tucson, AZ) AST-001 dual buffer video digitizer which interfaces directly with the ultrasound unit. The basic components of the system are shown schematically in Fig 1.

Currently, the software allows for the acquisition of TRUS images in a format of $512 \times 512$ eight bit pixels. The interface board has sufficient memory for two such images and, by including the live image, it is possible to work with three images simultaneously. The images may be saved to and recalled from hard disk storage. Two images may be compared using a blink comparator function which rapidly alternates between images. Image contrast can be enhanced in real time using a histogram stretch and slide algorithm. This capability is helpful in the delineation of the boundaries of the prostate in the images.

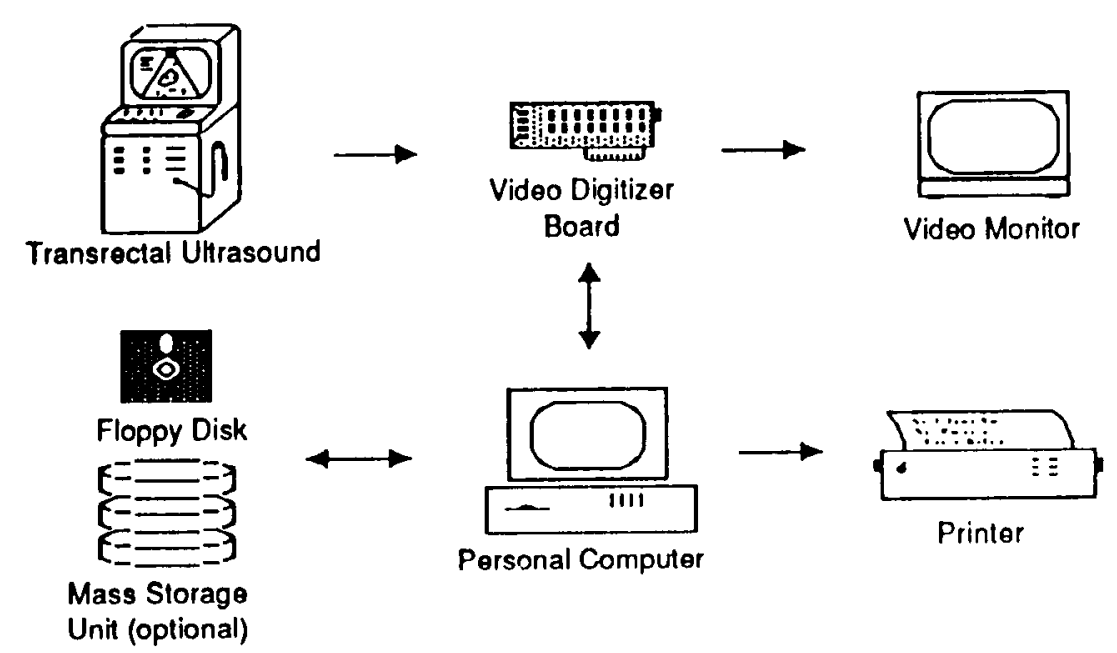

Fig 1. Digital transrectal ultrasound system schematic. 
We use a mouse to control the software in a point and select fashion. This type of interaction is one of the quickest and easiest types of interfaces to master; examples of menu selection options are shown in Fig 2.

The most important fcature of PROST is that it enables the user to easily measure the geometrical parameters described by Kimura et al. ${ }^{8}$ The software maintains a data sheet for each patient which is automatically filled in as the user makes the relevant measurements (Fig 3 ). The user performs these measurements directly on the prostate image by using the mouse to direct a cursor. Once the user has marked the perimeter and two diameters of the prostate, the software automatically enters the measurements into the data sheet. The user can easily remeasure if an error is made.

The software also keeps track of the images used and presents them to the operator in a serial fashion corresponding to the correct anatomical progression of the $5 \mathrm{~mm}$ slices. Alternatively, the operator may request to view an individual image.

In summary, this system provides a simple, efficient, and easily learned method for obtaining quantitative measurements and for performing necessary calculations to analyze TRUS images. Furthermore, the system may be easily modified through simple software updates to include new types of measurements and new analysis methods.

\section{Patients}

We obtained our patient base from a population in a retirement oriented community attending a private, singlesite urological practice. On the average, this practice performs about 15 to 20 transrectal ultrasound exams per month. Thirty-eight consecutive patients undergoing the indicated procedure were used in this study and all were evaluated during 1989. The patient ages ranged from 53 to 82 with a mean of 72 . The indications for the TRUS exam were a suspicious finding on physical exam, symptoms attributable to the prostate (hematoejaculate), and/or an elevated PSA

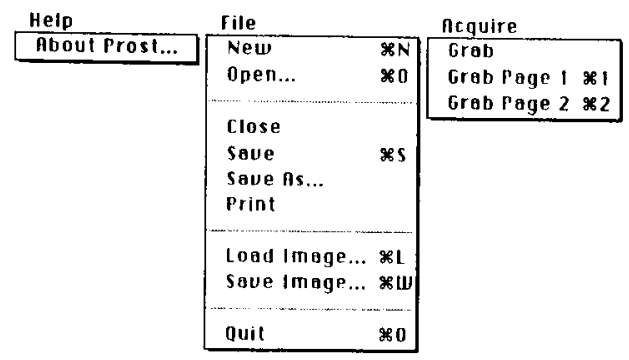

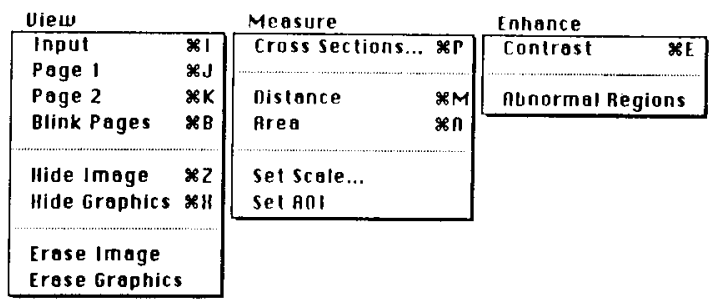

Fig 2. Examples of menu bars from PROST. These main menu selections are presented after being "pulled down" under mouse control. serum titer. We deleted four of the patients from the data set, including one patient who had had a transurethral prostatectomy previously and had distorted prostate anatomy. The data sets of the other three patients were lost because of technical reasons.

\section{TRUS Examination}

Patients were routinely placed in the lithotomy position and a Bruel \& Kjaer (Marlborough, MA) endosonic probe, Type $1850(7 \mathrm{MHz})$, was inserted rectally. The probe was attached to a stepping unit and withdrawn at $5 \mathrm{~mm}$ intervals. The ultrasound images at each level were obtained using a Bruel \& Kjaer Type 1846 ultrasound scanner.

At each level, we digitized and stored the ultrasound image in a microcomputer equipped with a Western Research Company, Inc (Tucson, AZ) video digitizer and the PROST software package. The image acquisition process did not modify the routine evaluation of the patient and had no impact on the medical or surgical treatment of the patient. We were able to analyze the images at a later convenient time.

We obtained at least three ultrasound-guided, needle biopsies from each prostate lobe (left and right). Our patient diagnosis is based on the histological examination of the biopsy.

\section{Data Analysis}

We analyzed the data using relative operating characteristic (ROC) techniques as described by Beck and Shultz. ${ }^{10}$ ROC analysis is a rapid, effective method to determine and compare the diagnostic utility of the measured parameters. The significance of the area under the ROC curves (AUC) was determined by using the standard critical ratio test. We used the two-sample $t$ test to calculate significance of the difference between the cancer and no-cancer group means. The raw data for each patient were maintained in a dBase III (Culver City, CA) data base, and we performed the analysis using a dBase command file designed to perform the ROC analysis and calculate the $z$ and $t$ values. The $z$ values are obtained for the standard critical ratio test following the method of Beck and Shultz ${ }^{j 0}$; these values are used in a look-up table to determine the associated probability, $\mathrm{p}$ (Table 2 and 3 ).

\section{RESULTS}

The parameters used in the analysis by Kimura et $\mathrm{al}^{8}$ were the anterior/posterior diameter, transverse diameter, and the perimeter of the prostate (Fig 4). These parameters provide the basic geometrical information needed to evaluate the characteristic changes, noted by Watanabe et al ${ }^{9}$ which occur with the prostatic cancer. From these values they calculated an asymmetry index (ASI), the anterior/posterior to transverse diameter ratio (ATR), a presumed circular area ratio (PCAR), a section dissimilarity index (DSI), and the glandular weight. The ASI, ATR and 


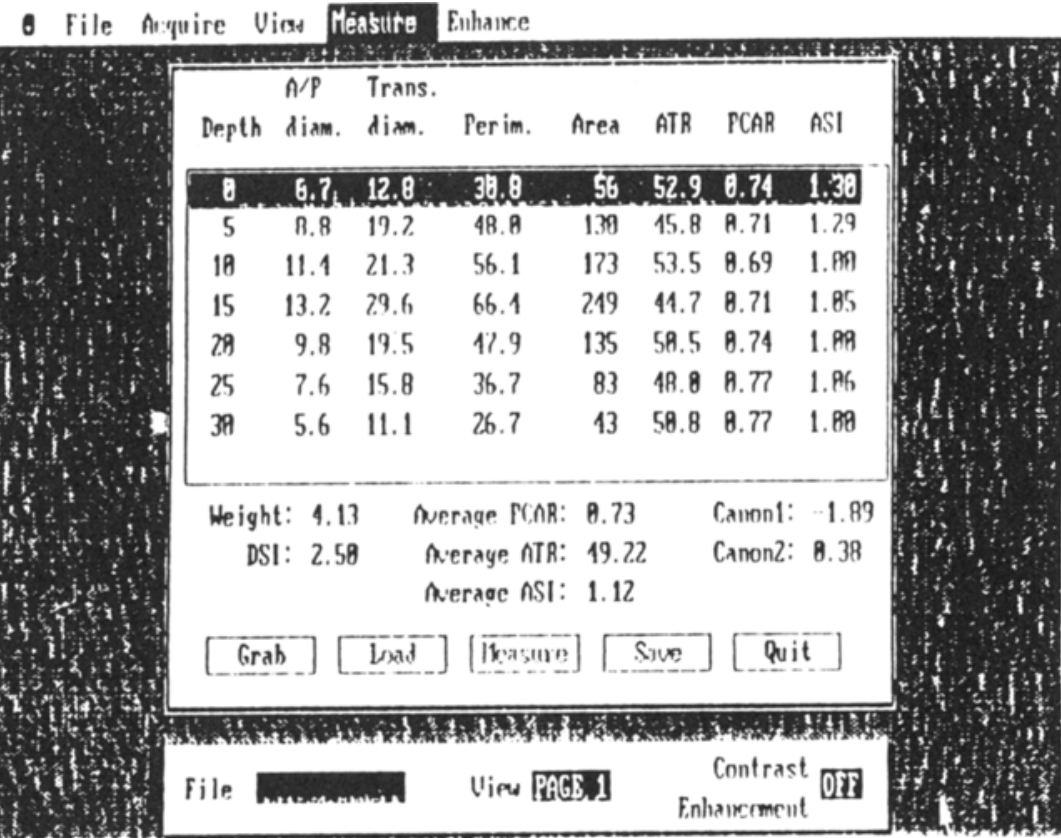

Fig 3. Data sheet presented during the measuring routine. This data window appears after solecting Cross Sections from the Measure menu. The geometric parameters are automatically entered into the appropriate spece after the user marks the necessery prostate features.
PCAR of only the largest section were used as parameters in the analysis. The DSI and weight were calculated using data from all sections.

The ASI is the maximum of the ratios of the halves of the transverse diameter (ie, maximum of $\mathrm{AX} / \mathrm{BX}$, or BX/AX as defined in Fig 4). The PCAR is the actual area divided by the area of a circle which has a circumference equal to the measured slice perimeter. Shapes nearly circular have a PCAR approaching one. The DSI is a measure of variations in shape of consecutive sections. It is calculated as the sum of absolute differences between the PCARs of adjacent sections weighted by the sum of the section areas;
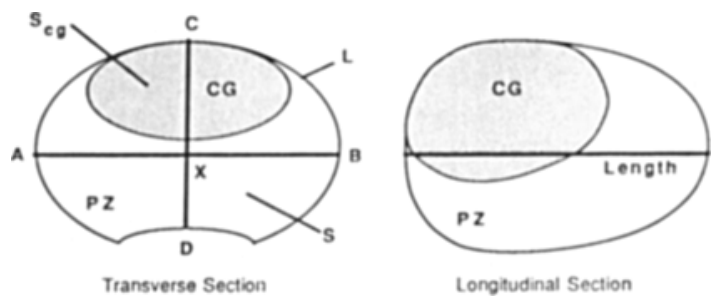

Fig 4. Anatomic features of prostate gland. This is a schematic of the transwerse and longitudinal view of a prostate gland upon which the various measured parameters are indicated. $L$ is the perimeter of the prostate section, $S$ is the section ares, $A B$ is the transverse axis, $C D$ is the anterior/posterior axis, $X$ is the point the axes cross. $S_{\mathrm{tg}}$ is the area of the central gland, $C G$ is the central gland. and $P Z$ is the peripheral zone. this causes the larger sections to contribute more to the DSI.

For each of the 34 patients in our study we determined these parameters, as well as the average PCAR, average ATR, and maximum ATR. The patients were assigned to either a cancer or no-cancer category based upon the biopsy results. The average of the measured values in each category are shown in Table 1 . Also shown are results of a two-sample $t$ test that indicates the significance of a difference between the mean values for the two categories.

The age of the patient, PSA level, and PCAR were all significantly greater ( $>95 \%$ confidence level) in the patients with cancer. The ATR,

Table 1. Comparison of Parameter Means

\begin{tabular}{lccr}
\hline \multicolumn{1}{c}{ Parameters } & Cancer & No Cancer & \multicolumn{1}{c}{$\mathrm{t}^{*}$} \\
\hline $\mathrm{n}$ & 14 & 20 & - \\
Age & $74 \pm 6$ & $70 \pm 6$ & 2.26 \\
PSA & $61 \pm 148$ & $4.50 \pm 4$ & 1.72 \\
PCAR & $0.82 \pm 0.05$ & $0.77 \pm 0.08$ & 2.02 \\
Weight & $42 \pm 22$ & $37 \pm 17$ & 0.69 \\
ATR & $58 \pm 9$ & $53 \pm 9$ & 1.50 \\
DSI & $4.1 \pm 1.8$ & $4.5 \pm 1.7$ & -0.69 \\
ASI & $1.1 \pm 0.04$ & $1.1 \pm 0.11$ & -0.71 \\
Maximum ATR & $76 \pm 20$ & $75 \pm 16$ & 0.08 \\
Average PCAR & $0.81 \pm 0.06$ & $0.79 \pm 0.07$ & 0.84 \\
Average ATR & $61 \pm 10$ & $60 \pm 8$ & 0.39 \\
\hline
\end{tabular}

*Reject hypothesis that the means are the same with $95 \%$ confidence if $|t|>t_{33,05}=1.69$. 
which is closely related to the PCAR, was also highly significant ( $>92 \%$ confidence level). None of the other parameters were significantly different between the two groups.

Figure 5 shows some representative ROC curves obtained from our ROC analysis of the data. In this figure the greater the area under the ROC curve, the greater the ability of the test to indicate patients with cancer. An area under the curve of 0.5 is found with tests that have no predictive value and this curve is shown as a diagonal line in Fig 5. Clearly, the best results are obtained when the PSA level, PCAR, or ATR are considered. The other parameters are of no value in diagnosing the presence of prostate cancer.

The results of the ROC analysis are summarized in Table 2 . We performed the critical ratio test on each curve to test for a significant difference from the value of 0.5 (ie, a test of no value). This analysis that the PSA, PCAR value, and the ATR value were all significantly different from the value of 0.5 at a significance greater than a $95 \%$ confidence level. The other values were not significantly different and were randomly associated with cancer in the prostate.

A pair-wise comparison of the area under the curves for different parameters was also performed, and these results indicate that curves for the ATR, PCAR, and PSA are statistically the same. This is in contrast to the comparison of the curves with, for example, the weight parameter which is statistically different (Table 3 ). Therefore, these data suggest that the parameters of

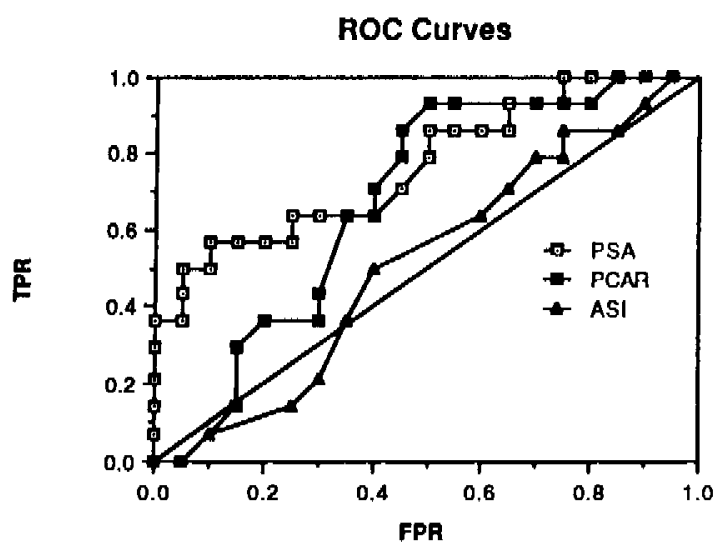

Fig 5. Reletive operating characteriatic curves for parameters measured from the TRUS exams and serum PSA levels. The diegonal line represents a curve with the AUC of 0.5 .
Table 2. Summary of ROC Analysis

\begin{tabular}{lccc}
\hline \multicolumn{1}{c}{ Parameter } & AUC & z & p \\
\hline PSA & 0.77 & 3.19 & 0.0003 \\
PCAR & 0.68 & 1.90 & 0.0287 \\
ATR & 0.67 & 1.79 & 0.0367 \\
Weight & 0.53 & 0.30 & 0.3820 \\
ASI & 0.52 & 0.16 & 0.4360 \\
DSI & 0.57 & 0.63 & 0.2640 \\
Maximum ATR & 0.51 & 0.05 & 0.4800 \\
Average ATR & 0.59 & 0.83 & 0.2030 \\
Average PCAR & 0.60 & 0.96 & 0.1690 \\
\hline
\end{tabular}

ATR, PCAR, and PSA are about equally efficacious in predicting the presence of prostatic cancer.

We constructed a four cell predictive value matrix for the significant parameters, and the calculated sensitivity, specificity, positive predictive value, and negative predictive value obtained are shown in Table 4. Values of PSA greater than $4.3 \mathrm{ng}$ per $\mathrm{ml}$ were considered abnormal. Inspection of the ROC curve for PCAR suggested that a cutoff of 0.75 would be close to optimal. Both the PSA and the PCAR measurement have high sensitivity ( $80 \%$ to $93 \%$ ) but relatively low specificity $(50 \%)$. The values we obtained for the serum PSA are very similar to those obtained by others. ${ }^{11}$ The two parameters are not significantly different in their ability to distinguish patients with cancer.

Interestingly, both PSA and PCAR have strong negative prediction potential ( $83 \%$ to $91 \%$ ). We have assumed that the two parameters (unlike PCAR and ATR) are unrelated. To determine if the PCAR could maintain a strong negative predictive potential regardless of the PSA value, we divided the population into those with PSA values greater than or less than 4.3. We then determined the negative predictive value of the PCAR measurement in each subgroup. The results show that, indeed, the negative predictive value was high in both: $80 \%$ in the patients with elevated PSA levels and $100 \%$ in those with normal levels. (Table 4).

Table 3. Statistical Comparison of the AUC of Pairs of Measurements

\begin{tabular}{lcc}
\hline Measurements & $z$ & $p$ \\
\hline PCAR, PSA & 0.96 & 0.169 \\
PCAR, ATR & 0.68 & 0.248 \\
PCAR, Weight & 2.58 & 0.005 \\
ATR, PSA & 1.02 & 0.154 \\
PSA, Weight & 2.60 & 0.005 \\
\hline
\end{tabular}




\begin{tabular}{|c|c|c|c|c|}
\hline $\begin{array}{c}\text { Positive } \\
\text { Diagnosis } \\
\text { Cniterta }\end{array}$ & Sensitivity & Specificity & $\begin{array}{c}1+1 \\
\text { Predictive }\end{array}$ & $\begin{array}{c}1-4 \\
\text { Predictive }\end{array}$ \\
\hline PSA $\mid>4.3\}$ & 80 & 50 & 55 & 83 \\
\hline PCAR (>0.76) & 93 & 50 & 57 & 91 \\
\hline PCAR $1>0.751$ & 92 & 40 & 67 & 80 \\
\hline \multicolumn{5}{|l|}{$\operatorname{PSA}(>4.3)$} \\
\hline $\begin{array}{l}\text { PCAR } 1>0.75) \\
(P S A<4.3)\end{array}$ & 100 & 70 & 40 & 100 \\
\hline
\end{tabular}

\section{CONCLUSIONS}

In this study, we have correlated morphological measurements from TRUS studies of the prostate gland and serum PSA levels with the histological diagnosis from multiple biopsies of a group of high-risk patients. Our results indicate that these measurements have significant diagnostic potential.

The morphological measurements we obtained were different in two major aspects compared with the results obtained by Kimura et $\mathrm{al}^{8}$ First, we found no significant difference in weight between patients with or without prostate cancer. Second, we found that the PCAR was significantly larger in the cancer patients and that it was highly correlated with the ATR (correlation coefficient of 0.994).

The reason for these differences is not known but may be partly due to the fact that we were dealing with an elderly population. Most of the patients in our study had large prostates. On the other hand, the strong correlation between the ATR and the PCAR that we see is what we would expect from the geometrical relationship of the two parameters.

While the measurement of the PCAR or ATR could be used as a sensitive screening test, the low specificity of the measurement would result in a large number of false positive results. It is possible that the more useful role of the PCAR measurement would be to utilize the negative predictive value of a measurement less than 0.75 to obviate the need to biopsy. This could potentially reduce the expense and morbidity of a prostate cancer screening program. The PCAR measurement may be particularly useful in this role since it seems to be independent of the serum PSA level. Therefore, it may be possible to avoid biopsy in some patients even if they have an elevated PSA level. However, we will need to confirm these results with a larger group of patients before the role of morphological measurements in patient management can be defined. For this reason, we are currently in the process of enlarging our patient database.

More recently, Hardeman et $\mathrm{al}^{12}$ have reported on the value of the ATR measurement in staging of disease. This study included pathological staging and radical prostatectomy. They found that, of those tumors that involved both halves of the prostate and had penetrated the capsule, $71 \%$ had an ATR greater than 0.8 . None of the tumors that were confined to the prostate had an ATR greater than 0.8 . While our study was not designed to address questions of staging, it is interesting to note that none of our patients had cancers that penetrated the capsule and none had an ATR greater than 0.8 . This is consistent with the results of Hardeman et al. ${ }^{12}$

The major advantage of using the morphometric measurements is that they are quantitative and reproducible. They do not depend on the subjective analysis of the individual performing the exam. Furthermore, the measurements are easy to perform and do not require highly trained personnel. The availability of this type of objective data could provide the basis for the development of expert systems to help physicians in the analysis of patients. The morphometric measurements may also help augment ultrasound studies of prostates that have isoechoic lesions (possibly occurring in as many as $37 \%$ of cases ${ }^{12}$ ).

We have demonstrated that images obtained from transrectal ultrasound studies can be easily and economically captured in a digital format. This enables computer-aided analysis of the information and provides flexibility to experiment with a wide range of software applications. Researchers do not usually have this capability, since most transrectal ultrasound instruments do not have provisions for creating new measurement and analysis functions. The system we have described in this paper exemplifies the potential capabilities for developing new methodologies for analysis of the prostate gland.

From our experience we believe this system offers researchers the ability to manipulate ultrasound images in an experimental fashion which 
may lead to improved diagnostic utility of TRUS. It also provides a means to maintain patient databases of TRUS images for future reference. The images could be used for comparison with future studies, for example to determine if there is progression in lesions being treated pharmacologically. A prostate image database also provides opportunities to develop effective computer- aided teaching and testing systems which could help train physicians to interpret TRUS images.

\section{ACKNOWLEDGMENTS}

We acknowledge the assistance of Bruel and Kjaer, Inc who kindly provided a TRUS unit and transrectal probes for use in this research; some critical elements of this research project were funded by grants from the National Cancer Institute of the National Institutes of Health.

\section{REFERENCES}

1. Cancer Statistics, 1988. CA 38:14-15, 1988

2. Baron $E$, Angrist A: Incidence of occult carcinoma of the prostate after 50 years of age. Arch Pathol 32:787.793, 1941

3. Andrews GS: Latent carcinoma of the prostate. J Clin Pathol 2:197, 1949

4. Walsh PC: Benign prostatic hyperplasia, in Harrion JH, Gittes RF, Perlmutter AD, et al (eds): Campbell's Urology (ed 4). Philadelphia, PA, Saunders, 1979, p 949

5. Killian CS, Enrich LJ, Vargas FP, et al: Relative reliability of five serially measured markers for prognosis of progression of prostate cancer. JNCI 76:179-185, 1986

6. MeNeal JE: Regional morphology and pathology of the prostate. Am J Clin Pathol 49:347-357, 1968

7. Lee F, Littrup PJ, Torp-Pedersen ST, et al: Prostate cancer: Comparison of transrectal US and digital rectal examination for screening. Radiology 168:389-394, 1988
8. Kimura A, Nakamura S, Niizuma M, et al: Quantitative analysis of ultrasonogram of the prostate. J Clin Liltrasound 14:501-507, 1986

9. Watanabe $H$, Igari $D$, Tanashi $Y$, et al: Transrectal ultrasonotomography of the prostate. J Urol 114:734-739, 1975

10. Beck JR, Shultz EK: The use of relative operating characteristic (ROC) curves in test performance evaluation. Arch Pathol Lab Med 110:13-20, 1986

11. Drago JR, Badalament RA, Wientjes MG, et al: Relative value of prostate-specific antigen and prostatic acid phosphatase in diagnosis and management of adenocarcinoma of prostate. Urology 34:187-190, 1989

12. Hardeman SW, Causey JQ, Hickey DP: Transrectal ultrasound for staging prior to radical prostatectomy. Urology 34:175-180, 1989 